\title{
Design of Intelligent Streetlights Monitoring Device Based on SMS of GSM
}

\author{
Guangli Long* \\ School of Physics and telecommunication Engineering, Shaanxi University of Technology, Hanzhong 723001, P.R. Chi- \\ na
}

\begin{abstract}
In order to save energy and for effective management of street lights, an intelligent streetlights monitoring device has been designed. The hardware part of the device is made up of MCU, GSM module, circuit of street lights, clock circuit, light controlling circuit, current detection circuit and so on. Software includes the main program, the subprogram for reading short message, subprogram for replying SMS, etc. The software is programmed with C language, and is compiled on the Keil platform. After the success, the .hex file is generated, which will be downloaded to the MCU. Experimental verification provides realization of the message to control the lights turn on and off. Also, the real-time clock can be realized to control the lights turning on and off, according to the sunlight to control the streetlamp. When the streetlamp is damaged, it can automatically alarm.
\end{abstract}

Keywords: Streetlights, GSM module, Short Message, Micro Controller Unit, Streetlamp. Automatic alarm

\section{INTRODUCTION}

Streetlight is a public facility necessary for the city. The degree of modernization of the city can be displayed from the lamp controlling mode and management level. There are three kinds of traditional streetlamp controlling modes: Manual controlling mode is pre-established schedule, in which the streetlamps are operated manually by the person on duty [1]. Time controlling mode basically opens or closes lights in the prescribed time with time. If needs to adjust, it can only be done by the manual operation. The light controlling mode takes the illumination as the basis. The streetlamps are opened or closed according to the light shading to control. If there is lack of light during the day or night glare, it may lead to malfunction. These three kinds of modes have no remote data acquisition and communication function. It cannot be timely to know whether lights on / off correctly or whether the work is normal or not. Also it can't be a rapid treatment, it can cause the waste of energy and accidents, and it cannot adapt to the needs of the development of city modernization [2]. The GSM Short Message [3, 4] is used as the communication mode of the monitoring system of city streetlamp. Management system can control the streetlamp working state and fault conditions through the mobile phone or PC machine, and the switch can be arbitrarily controlling streetlamp lighting. Through a convenient and quick processing of fault recovery, it can save a lot of human and material resources and improve work efficiency.

\section{SYSTEM PRINCIPLE}

The principle diagram of GSM short message intelligent streetlamp monitoring device is shown in Fig. (1).

The device is composed of controlling terminals of lamp detection and monitoring center. Controlling terminal of

\footnotetext{
*Address correspondence to this author at the School of Physics and telecommunication Engineering, Shaanxi University of Technology, Shaanxi, 723001, P.R. China; Tel: 13098208081; E-mail:1g120088@163.com
}

streetlamp detection device is composed of MCU as the core, the circuit of real-time clock, light sensor controlling circuit, current detection circuit, GSM module of GTM900C, keyboard, LCD screen, and the circuit of driving streetlamp. Among them the real time clock circuit controls time on the controlling detection terminal of street light to ensure time accuracy. Light sensing circuit automatically closes streetlamp in daytime and opens streetlamp in the night according to the different strength light of the day and the night. Current detection circuit mainly detects the current of controlling streetlamp to judge the damage. LCD screen mainly displays mode parameters for time controlling, light controlling or current of lamp. Keyboard mainly completes the relevant parameter settings. The circuit of driving streetlamp opens or closes the lamp respectively under the mode of time, light or debugging. The monitoring center mainly consists of PC, GSM (Global System for mobile communication) module GTM900C, large screen projectors, printers and other equipment components responsibly for the operation of the whole system. Of which: large screen projector can dynamically display working condition and operation parameters of each streetlamp detection controlling terminal. The streetlamp controlling personnel determine one of three modes according to time, light or debugging for on / off street light in PC. The data is monitored through the GSM module GTM900C. The alarm is recorded when the measured value and calculated value are beyond the permitted range.

\section{DESIGN OF THE HARDWARE}

\subsection{Design of MCU Controlling System}

MCU (Micro Controller Unit) is selected. MCU minimum system [5] consists of MCU STC89C52, clock circuit and reset circuit. The main functions are controlled by the module of scheduling. 


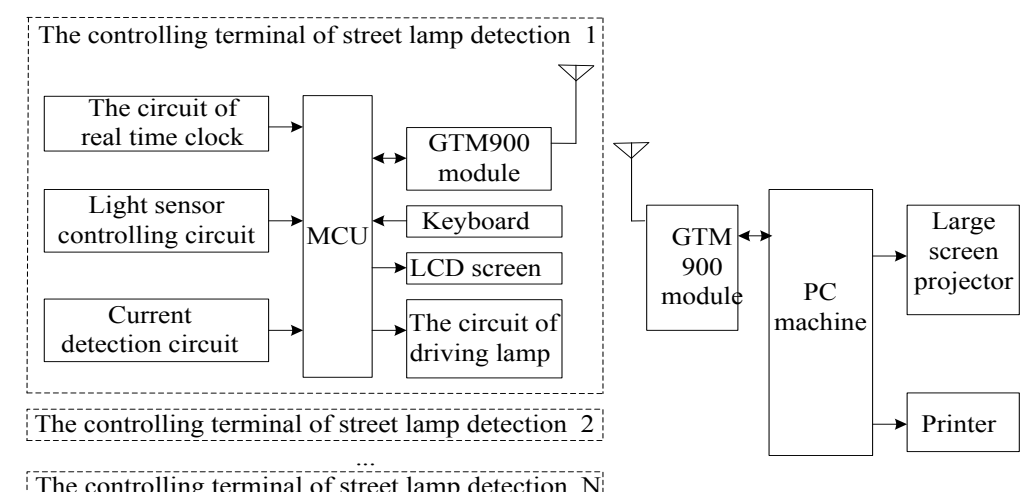

The controlling terminal of street lamp detection $\mathrm{N}$

Fig. (1). The principle diagram of GSM short message intelligent streetlamp monitoring device.

STC89C52 MCU is a 8 bit CMOS and low-power microcontroller. It is compatible with 51 series microcontroller. Its interior has $8 \mathrm{~K}$ bytes FLASH program storage space and 256 bytes of internal RAM, 32 programmable I/O interface, three 16 bit timer / counter, full duplex UART serial interface, built-in watchdog timer, double data pointer.

Reset circuit of MCU is generally divided into three kinds: manual reset, automatic power on reset, manual and automatic reset. The power on reset is achieved through a capacitor and a resistor in series. When the MCU system is electric, capacitor for charging takes time, a high level should be maintained at the RST end for some period of time. MCU completed reset after the capacitor charging because of the effects of RST resistance end recovery low level. The manual reset is achieved through a button and a resistor in series. When the button is pressed, it results in high end RST. When the button is released, RST terminal becomes low, and the MCU completes reduction. System resets circuit with manual and automatic reset modes. It not only has the automatic power on reset function, but also has a small button to manually reset.

MCU clock source is provided in two ways, one is the external clock source to provide the clock for the microcontroller, another is the internal clock chip. The internal clock mode is selected in the system clock circuit. An external crystal oscillator connects the MCU XTAL1 and the XTAL2 pins. $11.0592 \mathrm{MHz}$ crystal is selected. The internal clock frequency is $11.0592 \mathrm{MHz}$. Two $30 \mathrm{pF}$ ceramic capacitors are chosen in order to help the crystal oscillator, which is connected with the crystal legs in series.

\subsection{Huawei GTM900C Module}

Huawei GTM900C module and its peripheral circuit is shown in Fig. (2).

The GTM900C is a dual band $900 / 1800 \mathrm{MHz}$, highly integrated GSM/GPRS module, which is embedded with the TCP/IP protocol. Both the software and hardware are compatible with GTM900B, TC35i [6], MC39i. The AT command set is used in GTM900-C through the UART interface and the external CPU communication, which mainly realizes the wireless transmission and reception, baseband processing, audio processing and other functions. The keyboard, LCD and other external devices are controlled through an external CPU. Circuit interface connecting MCU is realized through ZIF (Zero Insertion Force) connector. The interface reads or sends the data of the GTM900C module, which is the core of GTM900C applications.

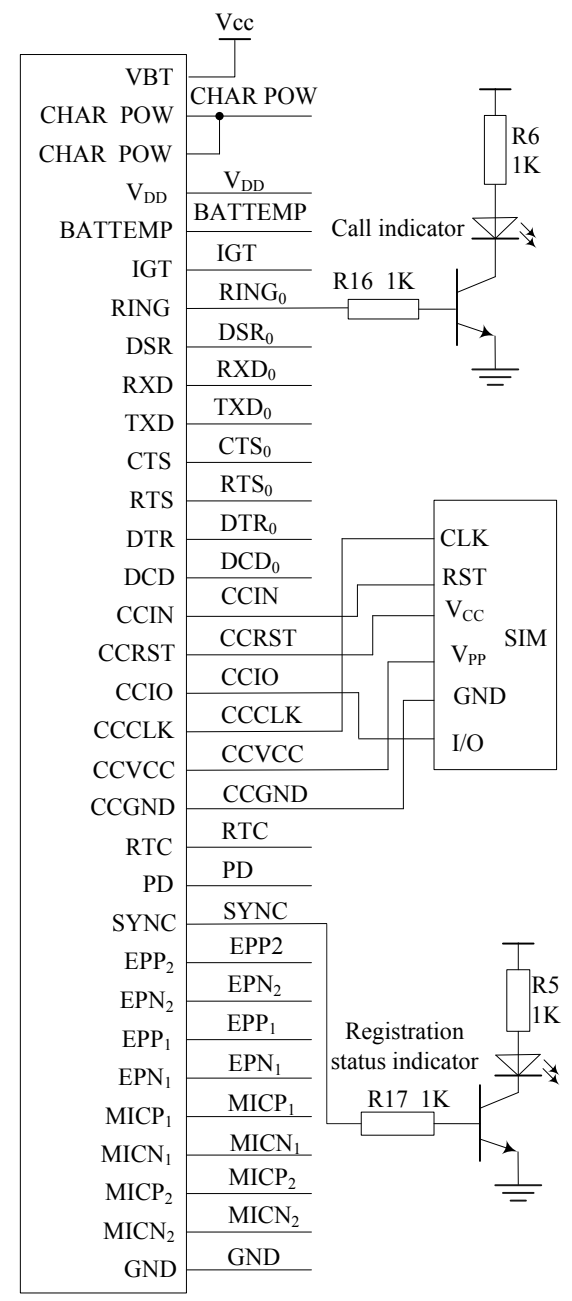

Fig. (2). Huawei GTM900C module and its peripheral circuit.

In Fig. (2), the GTM900C module 40 pins are led through a ZIF connector. These 40 pins can be divided into 5 categories: power supply, data input / output, SIM card, audio interface and controlling. No.16-23 pins are data input / 
output, respectively DSR0, RING0, RxD0, TxD0, CTS0, RTS0, DTR0 and DCD0. The TC35i module data input / output interface is actually a serial asynchronous transceiver, which conforms to the ITU-T standard RS232 interface. GTM900-C uses an external SIM card. No.24 29 pins are the SIM card pins. Connection modes of SIM card and GTM900-C are: CCIO, CCRST, CCCL, CCVCC and CCGND on the SIM card are connected directly through the SIM card reader and GTM900-C. CCIN pin of the ZIF connector is used to detect whether the SIM card is inserted well. If the connection is correct, then the CCIN pin output is high, otherwise the CCIN pin output is low level. If the amplitude range of input signal is more than the input signal of GTM900-C, then the input signal is needed for level adjustment. Output signal of GTM900-C module is TTL $2.85 \pm 0.1$ volt level interface, which is required to be converted when connected with the RS-232.

\subsection{The Design of Light Controlling Circuit}

Design of light controlling circuit is shown in Fig. (3).

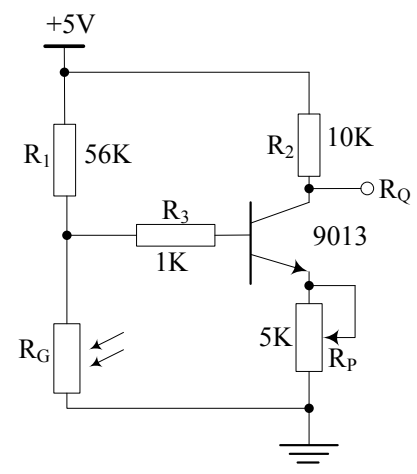

Fig. (3). Design of light controlling circuit.

Light sensing circuit is composed of photosensitive resistance RG, a triode S9013. The collector RQ of triode is connected with No.14 pins of MCU [7]. The output voltage of photosensitive total control is achieved through the MCU inputting a switching signal to control circuit of power supply, switching on and off of relay, and then the working situation of the whole circuit. Principle of work: the photosensitive resistor RG is reduced during the day because of strong light resulting in reduced voltage at both ends, so that the three tubes are at the reverse biased state output high level. The relay disconnection is controlled by MCU and the street- lamp is extinguished; the photosensitive resistor RG becomes large at night because the light is weak, so that the three tubes are at the saturated conduction state low level output. The relay opened attraction is controlled by the MCU and the streetlamp is opened.

\subsection{Design of Real Time Clock Controlling Circuit}

Design of real time clock controlling circuit is shown in Fig. (4). DS1302 is applied in the real-time clock, which is a real-time clock circuit of a high performance, has low power consumption and is embedded with RAM. It can be timed to the year, month, day, week, hours, minutes, seconds, and it has a leap year compensation function. The working voltage is $2.5 \mathrm{~V}-5.5 \mathrm{~V}$. A three wire interface is used for synchronous communication with $\mathrm{CPU}$, and the burst mode is used for transmission of multiple byte clock signal or RAM data. Power off protection circuit is composed of a button cell $3 \mathrm{~V}$ [8].

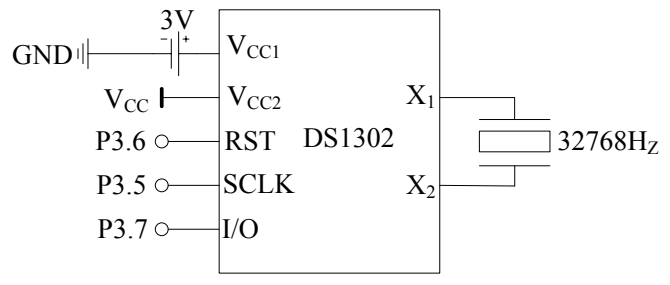

Fig. (4). Real time clock controlling circuit.

The circuit connection of DS1302: RST of DS1302 is connected with P3.6 of the MCU, which is a real-time clock reset pin ; SCLK of DS1302 is connected with the P3.5 of the MCU, which is a clock pin; I/O of DS1302 is connected with $\mathrm{P} 3.7$ of the MCU, which is a real-time clock data pin; $\mathrm{V}_{\mathrm{CC} 1}$ of DS1302 is connected to the $3 \mathrm{~V}$ button battery; $\mathrm{V}_{\mathrm{CC} 2}$ of DS1302 is connected to $5 \mathrm{~V}$ power supply; $\mathrm{X}_{1}$ and $\mathrm{X}_{2}$ of DS1302 are connected to $32768 \mathrm{~Hz}$ crystal.

\subsection{Design of Current Detection Circuit}

Design of current detection circuit is shown in Fig. (5).

Current detecting chip MAX471 is used, which is a production of precision integrated current sensor by an American MAXIM company. It is suitable for detection of current below $3 \mathrm{~A}$. The measuring precision is $+2 \%$ under high temperature conditions. MAX471 has a built-in current sensing resistor and a gain resistor. Its range is $3 \mathrm{~A}$, which allows

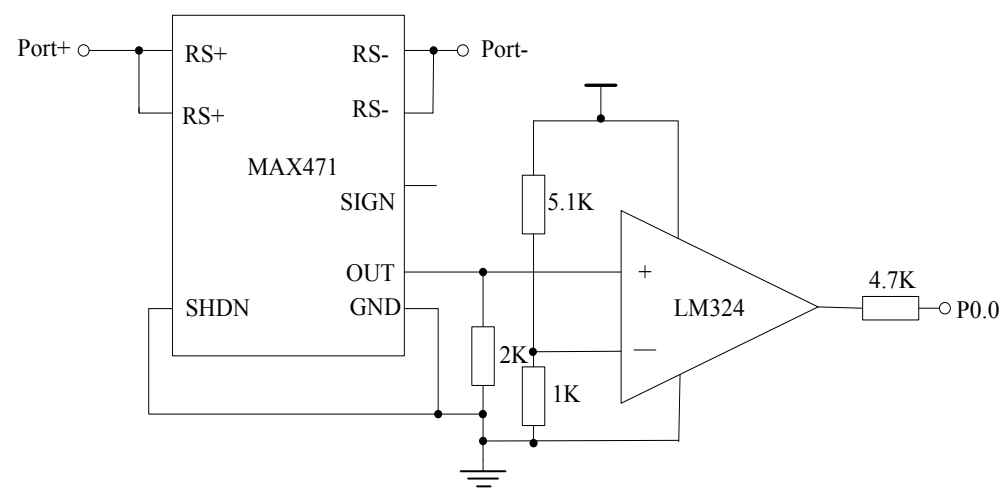

Fig. (5). Current detection circuit. 
multiple pieces of MAX471 to achieve a fixed amount of extended range [9].

MAX471 has two ends, RS+ and RS-, but no power end. However, the RS + can be replaced with the power end. SHDN is out of control terminal, and is connected with the ground in general at a high level so that the chip could enter in a power down mode. The supply current is rapidly reduced to $1.5 \mathrm{uA}$. Two pairs of RS+, RS- are respectively the positive and the negative terminals of the internal sense resistor. SIGN is polarity end. When the line current flows from the RS+ to the RS- terminal, SIGN terminal output is high level. On the other hand, when the current flows from RS- to the RS+ terminal, SIGN output is low level. OUT is the output end of the current, A resistor can be used to simply realize the current / voltage conversion for the reference point, and it can work in a wide voltage. OUT is proportional to the line current, the output impedance of the segment is $3 \mathrm{M} \Omega$. A $2 \mathrm{~K} \Omega$ resistor is connected between the out terminal and the ground and the output voltage proportional coefficient of MAX471 is $1 \mathrm{~V} / \mathrm{A}$.

LM324 is used in the comparative circuit of Fig. (5), and it includes four differential input operational amplifiers. Among them: "+" and "-" are in the two signal input terminal, "V+" and "V-" are in the positive and negative power terminal, and "Vo" is the output. In the two signal input, Viis the inverting input end, which represents the signal phase of the opamp output Vo and the input end is opposite; $\mathrm{Vi}+$ is the non-inverting input end, which represents the phase signal of the opamp output Vo and the input end is the same. When the voltage $\mathrm{Vi}+$ is greater than $\mathrm{Vi}-$, the output is high level in the circuit, otherwise, the output is low level, as shown in Fig. (5).

\subsection{Design of the Simulation Streetlamp Circuit}

Design of simulation streetlight circuit is shown in Fig. (6).

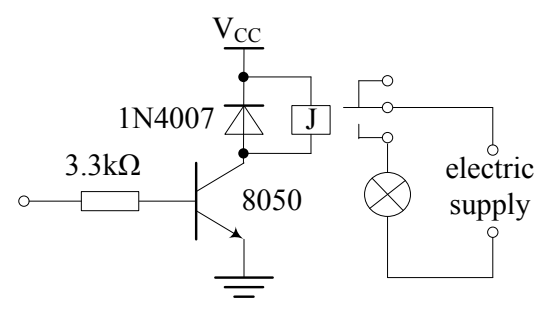

Fig. (6). Simulation streetlight circuit.

The triode may choose 9014 or $8050 ; 3.3 \mathrm{k} \Omega$ resistance is selected. When the triode conducts the high level of MCU $\mathrm{I} / \mathrm{O}$ port, the relay is attracted and the streetlamp is opened. On the contrary, when output from the MCU I/O port is low level, the streetlamps are extinguished. In order to eliminate the harmful effect of the sense of the relay coil EMF (electromotive force), suppression diode is reversed and connected to the relay coil, so that the electric potential is absorbed [10].

\section{DESIGN OF SOFTWARE}

The software is composed of the main program, read Short Message subroutine, SMS reply subroutine etc.

\subsection{Design of the Main Program}

Design of the main program flow chart is shown in Fig. (7). The main program starts the GSM module of GTM900C, the serial port and GTM900C initial after a delay of 10s. Preparation procedure is invoked for receiving SMS, receiving ready to be judged. Ready well, for receiving an SMS command; Otherwise, the judgment will send prepared, ready to reply message.

\subsection{Design of the Reading Short Message Subroutine}

Design of the reading short message subroutine flow chart is shown in Fig. (8). The operation procedure is executed, the content of the message is read to determine whether there is switch on/off relay instruction. A few lamps were judging the open, if the error is judged, the instruction flag is 1.

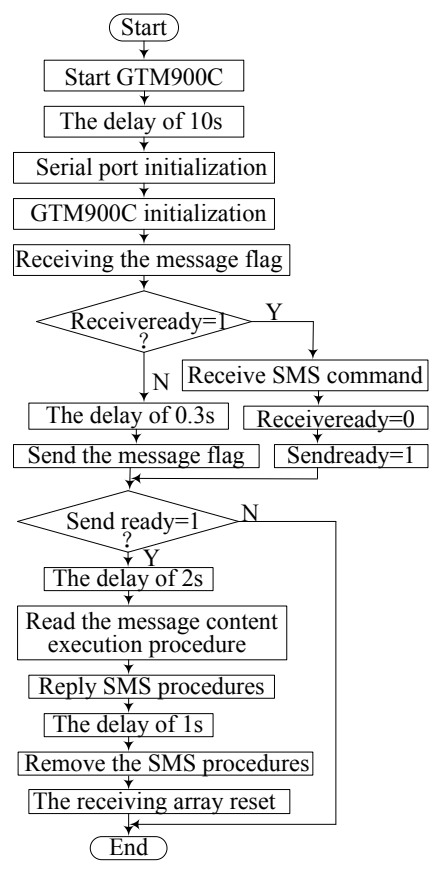

Fig. (7). The main program flow chart.

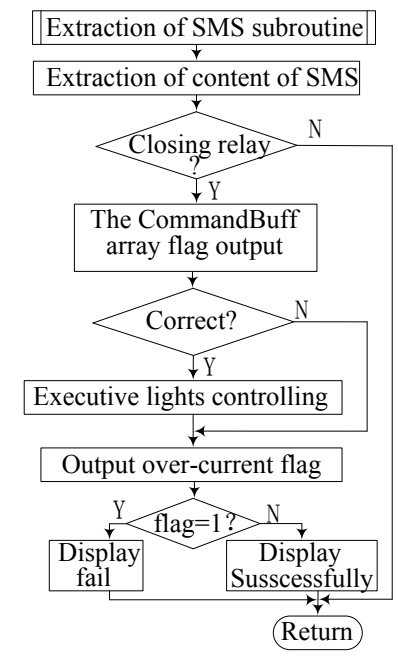

Fig. (8). The reading short message subroutine flow chart. 
First of all, the subroutine intercepts the instruction part of Short Message from the serial port sent with a statement, which was placed on the CommandBuf array [11]. Several flags (such as CommandBuf[0], CommandBuf[1], CommandBuf[2], CommandBuf[3] etc.) are judged. If the flag is correct, information for open and controlling objective is executed. If there are several flags, then program directly jumps to the last instruction, e.g. flag $=1$, which indicates that this controlling is failed. Overcurrent position is judged. If the flow is 1 , the program will jump to the end, or return. The instructions are operated with signs above the bits for the judgment. The case instruction is used through the output port of the MCU.

\subsection{Design of SMS Reply Subroutine}

Design of the SMS reply subroutine flow chart is shown in Fig. (9). Each SMS number is extracted from the reply message procedures, which determines the procedure flow. If there is overcurrent, simultaneously let SCM P3.6=0, send the "flow", otherwise, the instruction flag determines whether it is 0 . If flag $=0$, it indicates that the controlling operation is successful, then the reply is "success". If flag=1, it indicates that controlling operation is failed, then the reply is "fail" [12].

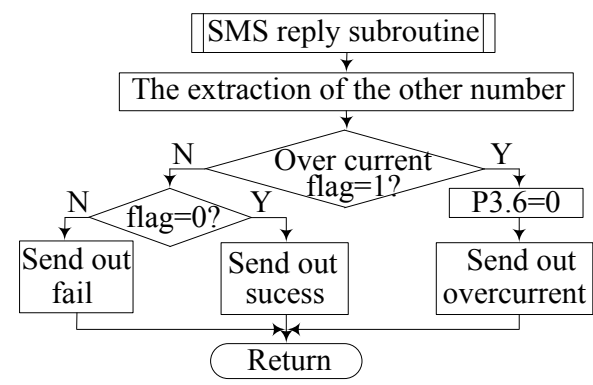

Fig. (9). The SMS reply subroutine flow chart.

The program is written using $\mathrm{C}$ language, and is compiled on the Keil platform [13]. When it is compiled successfully, hex file is generated. Using STC MCU download tools, the generated hex files are downloaded to the $\mathrm{MCU}$ STC89C52.

\section{THE EXPERIMENTAL RESULTS}

A cell circuit is simulated on the platform of Proteus [14]. After the success of simulation, PCB board is designed and made. The microcontroller of downloaded program and other components are welded on the PCB board, before its power function was tested. The GSM module transmits data and instructions under the control of the MCU. When MCU is at TTL level, a level conversion is needed. This level is converted by MAX232. LED lamp is operated by lamp circuit through relay. The monitoring center is replaced by mobile phone [15]. The SIM card is inserted into the card slot of GSM module. The following is the procedural message sequence: Power on, observation of GSM module lights in normal, the "open1" is sent to the GSM module for mobile phone, streetlamps light after $5 \mathrm{~s}$, and then a reply message is sent to the mobile phone "operate successfully!" after $2 \mathrm{~s}$. The "shut1" is transmitted to the GSM module for a mobile phone, the streetlamp turns off after $3 \mathrm{~s}$, and then the reply message is sent to the mobile phone "operate successfully!" after $2 \mathrm{~s}$. The above indicates that the test is a success.

\section{CONCLUSION}

This paper chooses GSM Short Message as a means of communication for completing automatic switching and lamp detection function. Hence, using this device, the labor intensity of streetlamp on manual patrol is solved. Besides, the automation level of monitoring streetlamp can be greatly improved and street lighting rate is increased. It is convenient for people and will generate good social benefits. If the device uses high-grade microprocessor and embedded system, it will reduce the short message delay, and further enhance the confidentiality and security, so that there will be a better and more extensive application.

\section{CONFLICT OF INTEREST}

The author confirms that this article content has no conflict of interest.

\section{ACKNOWLEDGEMENTS}

This paper is supported by the scientific research fund project of Shaanxi Province Education Office, project number: 2013JK1061; At the same time, this paper is supported by the science and Technology special plan of Hanzhoung city science and technology development, project number: 2013hzzx-45.

\section{REFERENCES}

[1] LI Ning, "Design and implement a remote terminal unit of the city street lamps".Baoding:North china electric power university,2008.

[2] CAI Weifeng, "The realization of wireless communication monitoring system for street lamps", Electric Automation, No. 5, pp.5962,2002.

[3] PAN Chao, LUO Xiaoqiao, HUANG Jia, "Design of smart home controlling system based on GSM SMS", Electronic Measurement Technology, vol. 36(6), pp. 121-124, 2013.

[4] BAI Yechao, YANG Bo, ZHANG Xinggan, “Design of intelligent door controlling safeguard system based on GSM module TC35", Electronic Measurement Technology, vol. 31(1), pp. 130-132, 144, 2008.

[5] LI Jianzhong, Principle and Application of the MCU. The second edition, Xi'an: Xidian University Press, 2008.

[6] ZHANG Hua, GAO Xu,"Sending and receiving short message by TC35 module", Computer Knowledge and Technology, vol. 6, No. 17, pp. 4817-4819, 4821, June 2010.

[7] YANG Zihua, HU Qian, LING Jiangrong, "Design and implementation of SMS system based on smartphone platform", Communications Technology, vol. 40, no. 09, pp. 70-72, 2007.

[8] JING Chunguo, SHU Dongmei, GU Deying, "Implementation of the times for sunrise and sunset algorithm in urban streetlight supervisory control system", Modern Computer, totally No. 163, no. 5, pp. 84-86, 2003.

[9] John Newbury, "Potential Communication Services Using Power Line Carriers and Broadband Integrated Services Digital Network", IEEE Transactions on Power Delivery, vol. 14, no. 4, pp. 11971121, 1999.

[10] LEI Weijia, WANG Huanhuan, XIE Xianzhong, "A relay selection algorithm based on channel information and residual energy in fountain relay transmission networks", Journal of Chongqing University of Posts and Telecommunications(Natural Science), vol. 24(2), pp. 190-195, 2012.

[11] LU Sheng, LI Zhengdai, "Short message receiving-sending system based on aT89S52 and GSM technology", Journal of Shanxi Agri- 
cultural University (Nature science edition), vol. 29(5), pp. 461465, 2009.

[12] SU Quanzhi, LI Chunsheng,REN Dianyi, etc., "Intelligentized monitoring management system of street lamps", Electrical Measurement \& Instrumentation, vol. 41(12), pp. 43-44, 20, 2004

[13] DUAN Xiangdong, WU Maosheng, "WU Yuzhi, Accurate delay methods of Keil C51 programming",Microcontrollers \& Embedded Systems, vol. 7(12), pp. 33-35, 2007.
[14] CHEN Chao,"Study on Protel DXP Packaging Components Library", Modern Electronics Technique, totally No. 311(24), pp. 163-167, 2009

[15] SU Lin, "Production of the anti-theft alarm listener by old mobile phone", Practical Electronics, totally No. 146(5), pp. 8-10, 2006.

Received: May 26, 2015

Revised: July 14, 2015

Accepted: August 10, 2015

(C) Guangli Long; Licensee Bentham Open.

This is an open access articles licensed under the terms of the Creative Commons Attribution-Non-Commercial 4.0 International Public License (CC BY-NC 4.0) (https://creativecommons.org/licenses/by-nc/4.0/legalcode), which permits unrestricted, non-commercial use, distribution and reproduction in any medium, provided that the work is properly cited. 